\title{
AN EXPERIMENTAL INFECTIVE ENDOCARDITIS MODEL IN RATS
}

\author{
Şükran Köse ${ }^{1}$, Tuba Tatlı Kış ${ }^{1}$, Canberk Yılmaz ${ }^{2}$, Mehmet Kış ${ }^{3}$, \\ Mustafa Barış ${ }^{4}$, Gülden Diniz ${ }^{5}$, Osman Yılmaz ${ }^{6}$
}

1 Department of Infectious Diseases and Clinical Microbiology, University of Health Sciences Tepecik Training and Research Hospital, Izmir, Turkey

2 Ege University School of Medicine, Izmir, Turkey.

${ }^{3}$ Department of Cardiology, Ege University School of Medicine, Izmir, Turkey.

${ }^{4}$ Department of Radiology, Dokuz Eylul University School of Medicine, Izmir, Turkey.

${ }^{5}$ Department of Pathology, Izmir Democracy University, Izmir, Turkey.

${ }^{6}$ Department of Laboratory Animals Science, Dokuz Eylül University School of Medicine, İzmir, Turkey.

Address for Correspondence: Tuba TATLI KIŞ, E-mail: tubatatlii@hotmail.com Received: 19.04.2021 Accepted: 20.05.2021 Available Online Date: 20.08.2021

(C) Copyright 2021 by Dokuz Eylül University, Institute of Health Sciences - Available online at https://dergipark.org.tr/en/pub/jbachs

Cite this article as: Kose S, Tatli Kis T, Yilmaz C, Kis M, Baris m, Diniz G, Yilmaz O. An experimental infective endocarditis model in rats. J Basic Clin Health Sci 2021; 3: 93- 99.

\begin{abstract}
Objective: Infective endocarditis (IE) is defined as infection of the endocardial surface of the heart. Updates are needed in the diagnosis and treatment of IE, as well as in animal models of IE. Based on this need, a new model of infective endocarditis induced by $S$. aureus was described in our study.

Methods: This study was performed on 7 Wistar albino male rats, each aged six months and weighing 250-300 g. Underwent the surgical implantation of a $20 \mathrm{G}$ catheter, to gain access to right common carotid artery. Twenty-four hours after implantation, $0.5 \mathrm{ml} 100.000$ colony forming unit (cfu) of S. aureus was injected via the tail vein and 3 days later echocardiography was performed and rats subsequently sacrificed. IE was later diagnosed histopathologically.

Results: Two of the rats were exitus one day after $S$. auerus was given. The mortality rate of the experiment was $28.5 \%$. Histopathological examination revealed vegetations and bacterial colonization were detected in the endocardium in all rats that protruded from the endocardium to the cardiac cavity.

Conclusion: Our study is the first study in the literature to identify the IE rat model using the $20 \mathrm{G}$ catheter. Due to the practical application of the surgical procedure (use of $20 \mathrm{G}$ catheter) in our study, we think that it will provide much convenience to the researchers in the experimental research on IE diagnosis and treatment.
\end{abstract}

Keywords: Infective endocarditis, MSSA, rat, model

\section{INTRODUCTION}

Infective endocarditis (IE) is defined as an infection of the endocardial surface of the heart. Although cardiac valves (native/prosthesis) are involved most commonly, the mural endocardium, septal defects, or intracardiac devices (permanent pacemaker and/or defibrillators) may also be included. Despite IE is rare, it is still important because of the difficulty in diagnosis and treatment, as well as high mortality and morbidity (1). In-hospital mortality has been reported to be $20 \%$ in Europe or America and $30 \%$ in Turkey $(1,2)$. The causative agents often include Staphylococcus 
aureus, viridans streptococci, enterococci, coagulase negative staphylococci, and HACEK Group bacteria (Heamophilus aprophilus, Actinobacillus actinomycetemcomitans, Cardiobacterium hominis,

Eikonella corrodens, Kingella kingea). S.aureus is the most common cause of IE in developed countries. In recent years, the incidence of IE due to $S$. aureus has increased (3). In Turkey, $S$. aureus is the most common causative microorganism found in patients with IE (4).

The pathophysiology of IE includes endocardial damage, hypercoagulability, and bacteremia. The experimental IE models described to date are based on this pathophysiology (5-12). Animal models are widely used to investigate new diagnostic methods in IE and to evaluate the efficacy of various antimicrobial agents. Therefore, the described model should be an easily applicable and cost-efficient model with low complication risk. In animal models described to date, a polyethylene tube extending from the carotid artery or the jugular vein to the cardiac valve was primarily inserted into the vessel to induce endocardial damage. As in the pathophysiology of IE, platelet and fibrin deposition in the damaged area was ensured following the damage of endocardium hitting the polyethylene catheter with each movement of the cardiac valves. This tissue is called non-bacterial thrombotic endocarditis (NTBE) (13). This structure is a tissue to which microorganisms attach more readily than intact endocardium (13). Organisms such as $S$. aureus, C. albicans, and Enterococcus faecalis, which have fibronectin receptors on their surface, have been reported to attach more easily to NTBE structure (13). Therefore, after the formation of NBTE, IE has been produced by injecting the microorganisms with the fibronectin receptor on the surface such as $S$. Aureus, C. Albicans, Enterococcus faecalis into the circulatory system in experimental animal models (5-12).
In the surgical procedure stage of the previously described IE animal models, an incision was made in the vessel to insert the polyethylene tube into the vessel and the polyethylene tube was tried to be inserted into the vessel. This procedure may have a high complication rate such as bleeding risk and mortality rate. This study was planned based on the idea that whether IE may be produced in rats using a 20 gauge $(G)$ catheter which is available and readily obtainable in all clinics instead of the polyethylene tube used in the surgical procedure stage. Using the catheter and ensuring the entry in the vessel at once without having an incision to insert the tube into the vessel will reduce the risk of complications in an animal model which already has a high risk of complications such as bleeding and mortality and will also provide ease of supply.

Updates are needed in the diagnosis and treatment of IE, as well as in animal models of IE. Based on this need, a new model of IE induced by $S$. aureus was described in our study to present a new approach to the difficulties encountered during surgical procedures in older models, which has not been previously described in the literature.

\section{MATERIAL AND METHODS}

This study was performed on 7 Wistar albino male rats, each aged six months and weighing 250-300 g. Before starting the study, the application no 40/2017 was done and ethical approval was obtained from the Experimental Animals Local Ethical Committee of Dokuz Eylül University, Turkey. All the rats were housed in standard rat chambers and cages with a dark/light period of 12/12 hours and relative humidity of $50-60 \%$ at room temperature of $20-22^{\circ} \mathrm{C}$ until the trial was terminated. They were fed ad libitum with standard rat feed and water. During the trial, daily weight, feed and water consumption per cage, and rectal temperature were monitored.

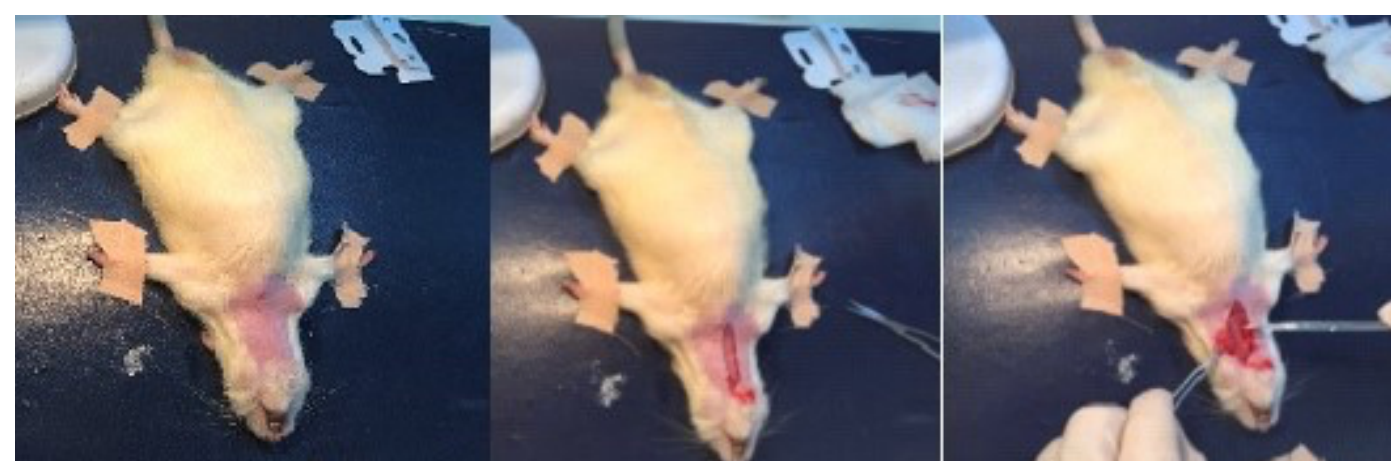

Figure 1. Dissection of subcutaneous tissues after preoperative shaving and the vertical incision. 


\section{Surgical procedure}

Preoperative ketamine $35-45 \mathrm{mg} / \mathrm{kg}+$ xylazine $5-10$ $\mathrm{mg} / \mathrm{kg}$ was administered intraperitoneally (I.P.) for anesthesia. The neck area from under the chin to the bottom of the rib cage was shaved after cleaning with $10 \%$ povidone-iodine. A vertical midline skin incision was made on the neck, skin and subcutaneous tissues were passed. The sternothyroid, sternohyoid and sternocleidomastoid muscles were dissected (Shown in Figure 1).

After exposing the right common carotid artery and vagus nerve, the fascia between the vagus nerve and common carotid artery was dissected. The vagus nerve was released, the carotid artery was isolated (shown in Figure 2). The catheter team localization was determined as the junction of cranial thoracic aperture and common carotid artery. The common carotid artery was entered at the proximal of the clavicular bone.

The distal portion of the common carotid artery was ligated with 3.0 silk sutures for bleeding control (shown in Figure 3). After a 20-gauge catheter was inserted into the carotid artery and the steel guidewire was removed, the plastic part was advanced towards the aortic valve in the direction of the heart (shown in Figure 4). After touching the aortic valve, the outer part of the plastic was blunted by burning and separated from the other plastic portion of the catheter. Thus, blood flow out of the cardiovascular area was prevented. The catheter part which was in the vessel was fixed to the vessel with 3.0 silk sutures. After the control of bleeding around the catheter, muscle tissue, skin, and subcutaneous tissues were repaired (shown in Figure 5). The rat was left in the cage and $5 \mathrm{mg} / \mathrm{kg} /$ day xylazine was administered subcutaneously for postoperative

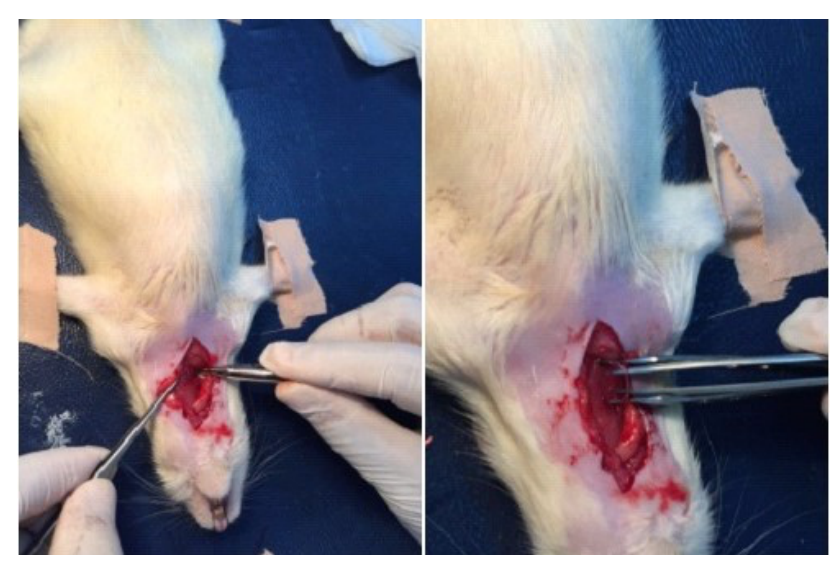

Figure 3. Isolation of the carotid artery.

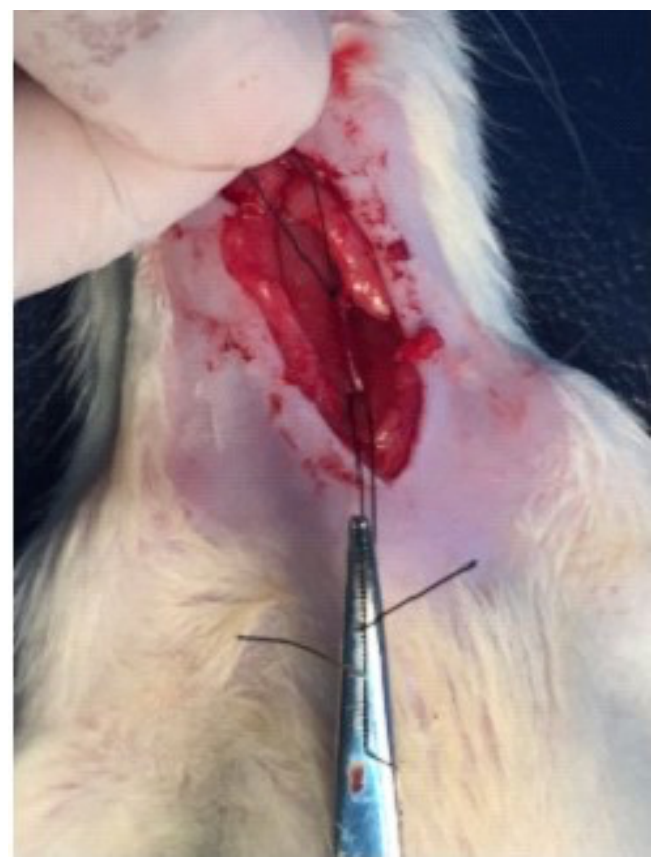

Figure 2. Ligation of the distal portion of the common carotid artery with 3.0 silk sutures.

analgesia following the surgical procedure was completed.

\section{Injection of Methicillin-Susceptible $S$. aureus (MSSA) into rats}

$10^{5}$ colony forming units (CFU) S. aureus ATCC 29213 strain (6) was given in $0.5 \mathrm{ml}$ of saline for each rat from the tail vein 24 hours after the surgical procedure is completed (shown in Figure 6).

\section{Performing Echocardiography in rats}

Echocardiography was performed using Visual Sonics, Vevo 2100 Micro-Ultrasound and small animal imaging system device as a non-invasive imaging method for the detection of vegetation on aortic valve 3 days after $S$. aureus injection.

\section{Microbiological examination}

The rats were sacrificed with high dose ketamine xylazine following echocardiography, 3 days after S. aureus injection. A median sternotomy incision was made. The heart was dissected from the sternum. The aortic valve was made visible by an incision in the left ventricle of the heart. After macroscopic control of the catheter and vegetations, the microbiological examination of vegetations tissue was performed. Vegetations are weighed and homogenized under sterile conditions. S.aureus growing per gram of tissue calculated as CFU/g 


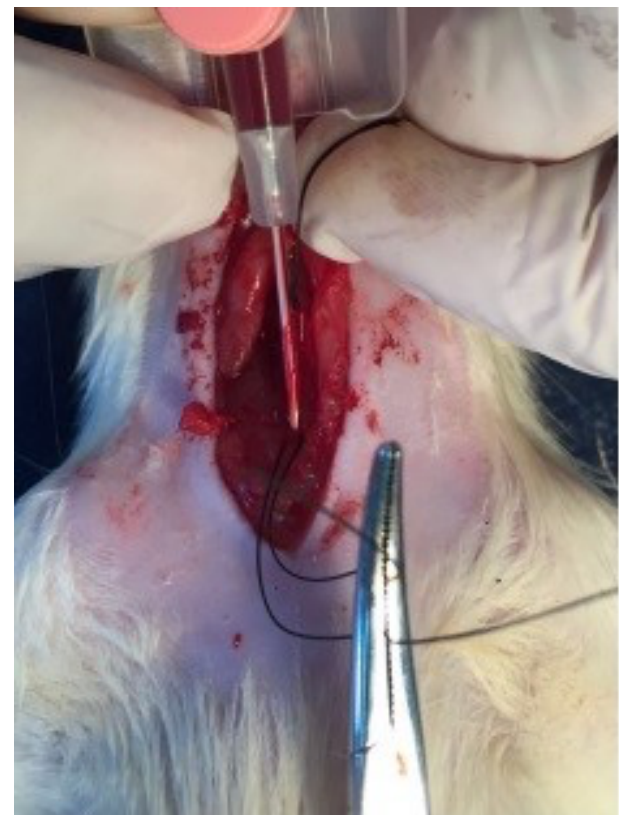

Figure 5.Introduction to the common carotid artery with a $20 \mathrm{G}$ catheter.

namely tissue quantitative culture was done. The culture antibiogram was performed to understand that the same S.aureus grew in vegetation tissue.

\section{Histopathological examination}

The tissue pieces were fixed in formalin and embedded in paraffin blocks after the follow-up and 4-5 $\mu \mathrm{m}$ sections were taken. The sections were stained with hematoxylin-eosin. The presence of endocardial polymorphonuclear leukocyte (PMNL) infiltration, edema, fibrin, and bacteria were evaluated in multiple sections.

\section{RESULTS}

Two of the rats died one day after injecting $S$. aureus. The mortality rate of the trial was found to be $28.5 \%$. A rectal temperature of $38.4{ }^{\circ} \mathrm{C}$ was found in one rat during daily rectal temperature monitoring.

\section{Microbiology}

In quantitative culture made from vegetation tissue traced in the hearts of rats, $104 \pm 0.53$ (Mean \pm SD) $\mathrm{CFU} / \mathrm{g}$ S.aureus was grown. As a result of the culture antibiogram, it was determined that the S.aureus that grew in the heart tissue after the model was formed was the same as that given to rats.

\section{Histopathology}

Histopathological examination revealed bacterial colonization and vegetations protruding from the

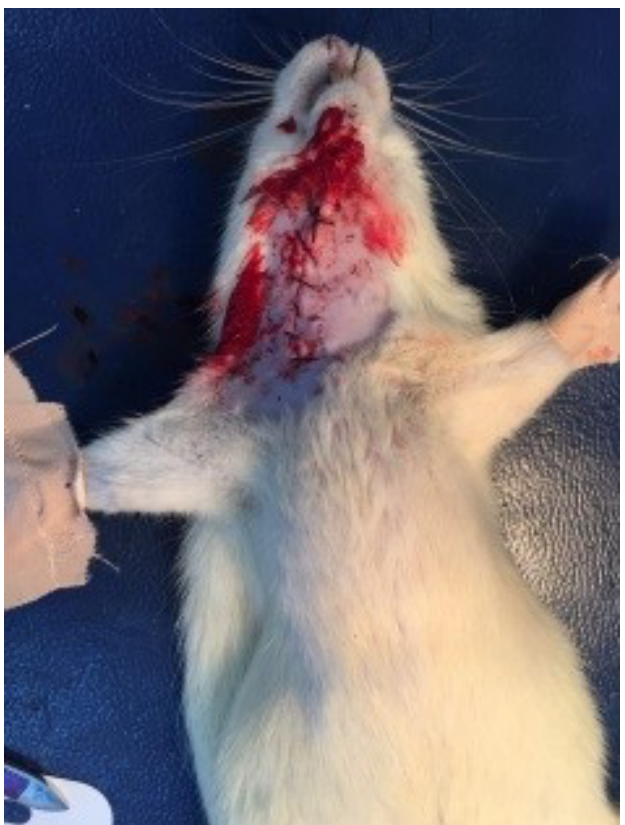

Figure 4. Skin subcutaneous tissue repair after surgery.

endocardium to the cardiac cavity in all rats (These lesions are shown in Figure 7). All rats were histopathologically diagnosed with IE. Myocarditis accompanied in one of the rats $(14.2 \%)$ and pneumonia in two $(28.5 \%)$. The renal examination of two rats $(28.5 \%)$ showed microabscess foci. The liver was unremarkable except for sinusoidal dilatation and Kupfer cell hyperplasia. The spleen was normal other than congestion and white pulp hyperplasia.

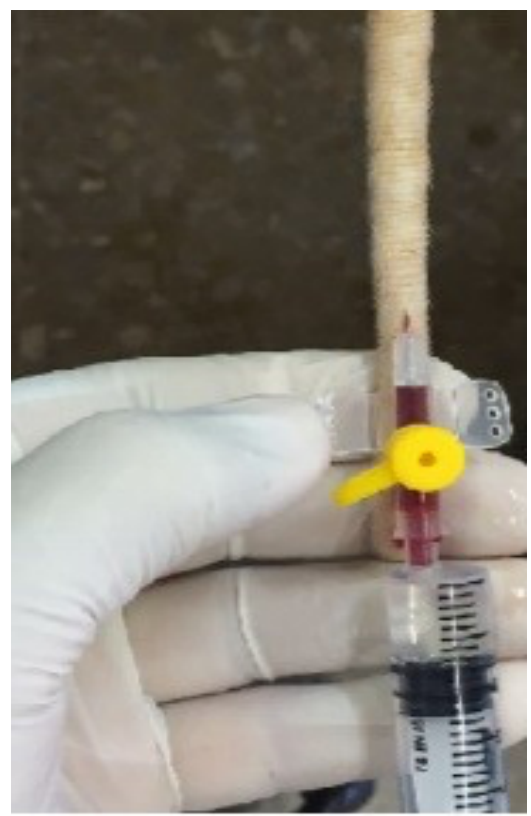

Figure 6. S. aureus injection from the tail vein. 


\section{Echocardiography}

Echocardiographic images from four rats randomly selected showed no echogenicity of vegetation on the aortic or mitral valve in any of the rats. The catheter placement was confirmed by echocardiography. The catheter had an appropriate location in all rats. The catheter tip of the rats revealed a thrombus with a size of $0.9^{\star} 0.8 \mathrm{~mm}, 2.8^{\star} 1.6 \mathrm{~mm}, 2.1^{*} 6.5 \mathrm{~mm}$, and $1.6^{\star} 2.1$ $\mathrm{mm}$, respectively. In a rat, thickening of the posterior right lateral wall of the ascending aorta reaching 1.7 $\mathrm{mm}$ and soft tissue increase around the vessel in the craniocaudal portion of the ascending aorta, extending up to approximately $8 \mathrm{~mm}$ were observed (aortitis). The aim of performing echocardiography in rats was to support the diagnosis by a non-invasive imaging method before sacrification.

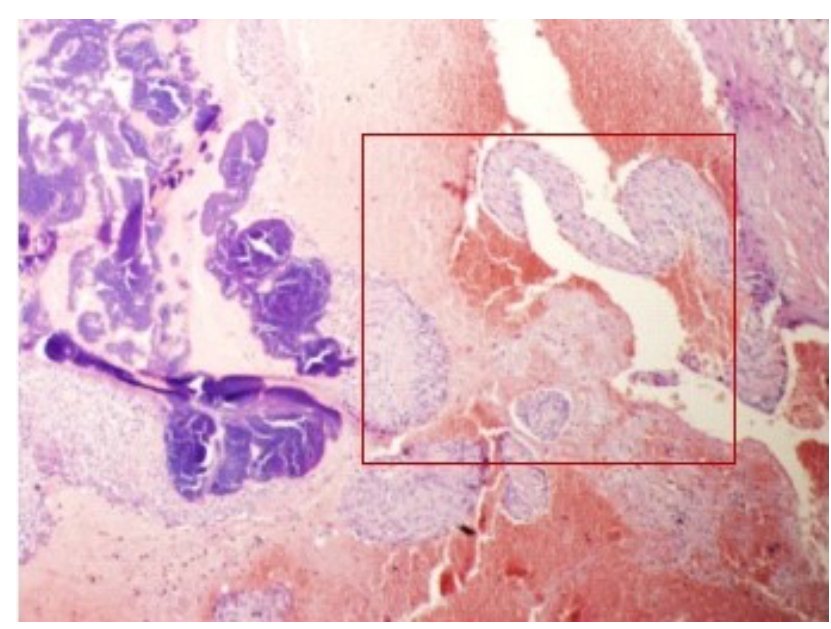

Figure 7. Endocardial vegetations protruding from the endocardium to the cardiac cavity.

\section{DISCUSSION}

In our study, the use of the $20 \mathrm{G}$ catheter during the surgical procedure of the IE rat model we created was defined for the first time in the literature. This study is important because it brings ease of application and updates to the IE rat models.

Various models of experimental IE have been described to date (5-12). This study has two important differences from the predefined IE animal models. Firstly, $20 \mathrm{G}$ catheter was used instead of the polyethylene tube in the surgical procedure for ease of application. The use of the catheter will be very convenient for researchers and it is important in terms of providing easier application than the polyethylene tubes. Other advantages of using a $20 \mathrm{G}$ catheter include the lower risk of bleeding and the lower postop fluid deficit of the animal. Another difference in our study from the other IE models described to date is that echocardiography was performed on rats before sacrification.

The use of polyethylene tubes in IE models dates back to old times. In one of the oldest studies on the IE model in the 1970s, the rabbit was used to create the model, a polyethylene tube was inserted into the external jugular vein and vegetation was formed on the tricuspid valve (5). However, the use of rats in IE models is advantageous compared to rabbit models because of being maintained more easily. However, in the following years, the IE model was tried in rats and the polyethylene tube was inserted into the carotid artery and vegetation was formed on the aortic valve (6). Today, we still see that the polyethylene tube is used in experimental rat studies for the diagnosis and treatment of IE (7). However, the use of polyethylene tubes has some disadvantages. While polyethylene tube is used during the introduction to the common carotid artery, an incision should be made on the surface of the artery. After the incision is made, the polyethylene tube with the blunt end is tried to be advanced towards the heart. Vasoconstriction occurs with the activation of the sympathetic nervous system on the surface of the artery during the incision of the artery, which causes difficulties in the insertion and advancement of the polyethylene tube into the artery. On the other hand, it is possible to enter the artery without any incision while using the $20 \mathrm{G}$ catheter. Entering the artery without vasoconstriction during catheter use reduces the risk of complications/bleeding, the post-op fluid requirement of the rat, the mortality rate during the experiment due to the surgical procedure and it provides quite convenience to the researchers. Therefore, compared to the studies performed with the polyethylene tube, the IE model with the catheter is important as it may be a pioneer for future studies on IE concerning complications and low mortality.

The second difference that distinguishes our study from other IE animal models is that echocardiography was performed on subjects prior to sacrification to support the diagnosis of endocarditis. The diagnosis was made histopathologically after sacrificing the animal in previous IE models. (14). In our study, histopathology was also used as the gold standard diagnostic method. Differently, echocardiography was performed on rats before sacrification. However, echocardiography showed no vegetation on the aortic or mitral valve. Echocardiography mostly helped to confirm the catheter location. The echocardiographic 
examination is still the most widely used imaging modality in the diagnosis of IE. Transthoracic echocardiography (TTE) and transesophageal echocardiography (TEE) have a complementary role in the diagnosis of IE (15). Echocardiography allows the diagnosis of IE in the majority of cases of vegetation, abscess, valve perforation, or reseparation of the artificial valve (15). However, echocardiography may not yield results in cases of IE with minor or no vegetation. In our study, echocardiography revealed no vegetation. Echocardiography was helpful in verifying the catheter tool location. The definitive diagnosis of IE was made by histopathology.

Based on the pathophysiology of IE, microorganisms with the fibronectin receptor on the surface (such as MSSA, MRSA, streptococcus spp, c. albicans) have been used in experimental endocarditis models (512). The reason for using $S$. aureus in our study is the increase of $S$. aureus-related IE along with the increase of health-related IE in recent years (3). S. aureus was used in our study with the idea that $S$. aureus-related IE studies will be needed more because of its incidence. However, we suggest that other microorganisms with the fibronectin receptor on the surface can be used in the IE rat model using a similar surgical procedure.

\section{CONCLUSION}

Our study is the first study in the literature to identify the IE rat model using the $20 \mathrm{G}$ catheter. There are two important differences from the predefined IE models of this study. The first is the introduction of a $20 \mathrm{G}$ catheter instead of a polyethylene tube into the common carotid artery during the surgical procedure, and the second is performing echocardiography in the rats as an imaging method before sacrification. The endocarditis rat model we described is a cost-efficient and easily applicable new model with low complication risk that may be a pioneer for future experimental animal studies on IE.

Conflict of interests: The authors declare that there is no conflict of interest.

Funding: This study was not supported by any organization.

Ethical approval : This study received the approval from the Dokuz Eylül University, Animal Local Ethics Committee with the protocol number 40/2017.

Peer-review: Externally peer-reviewed.

\section{REFERENCES}

1. Şimşek Yavuz S. Infective Endocarditis: An Update. Klimik Journal. 2015; 28(2): 46-67.

2. Cahill TJ, Prendergast BD. Infective Endocarditis. Lancet. 2016; 387: 882-93.

3. Pant S, Deshmukh A, Mehta JL. Reply: Trends in Infective Endocarditis: Incidence, Microbiology, and Valve Replacement in the United States From 2000 to 2011: The Devil Is in the Details. Journal of the American College of Cardiology. 2015 8; 66(10):1202-3.

4. Şimşek Yavuz S, Şensoy A, Kaşıkçıoğlu H, et al. Infective endocarditis in Turkey: aetiology, clinical features, and analysis of risk factors for mortality in 325 cases. International Journal of Infectious Diseases. 2015 Jan;30:106-14.

5. Durack DT, Beeson PB. Experimental bacterial endocarditis. I: colonization of a sterile vegetation. British Journal of Experimantal Pathology. 1972; 53(1): 44-9.

6. Fogarasi M, Pullman J, Winnard P Jr, Hnatowich DJ, Rusckowski M. Pretargeting of bacterial endocarditis in rats with streptavidin and $111 \mathrm{In}$ labeled biotin. J Nucl Med. 1999 Mar;40(3):48490.

7. Victorio GB, Bourdon LMB, Benavides LG, et al. Antifungal activity of caspofungin in experimental infective endocarditis caused by Candida albicans. Mem Inst Oswaldo Cruz. 2017; 112(5): 370-375.

8. Hady WA, Bayer AS, Xiong YQ. Experimental endocarditis model of methicillin resistant Staphylococcus aureus (MRSA) in rat. Journal of Visualized Experiments 2012; (64): 3863.

9. Que YA, Haefliger JA, Piroth L, et al. Fibrinogen and fibronectin binding cooperate for valve infection and invasion in Staphylococcus aureus experimental endocarditis. J Exp Med. 2005 May 16;201(10):1627-35.

10. Héraïef E, Glauser MP, Freedman LR. Natural history of aortic valve endocarditis in rats. Infect Immun. 1982 Jul;37(1):127-31.

11. Tran TT, Tam VH, Murray BE, Arias CA, Singh $\mathrm{KV}$. Efficacy of Telavancin Alone and in Combination with Ampicillin in a Rat Model of Enterococcus faecalis Endocarditis. Antimicrob Agents Chemother. 2017 May 24;61(6) :0248916. 
12. Miller WR, Singh KV, Arias CA, Murray BE. Adjunctive Clavulanic Acid Abolishes the Cefazolin Inoculum Effect in an Experimental Rat Model of Methicillin-Sensitive Staphylococcus aureus Endocarditis. Antimicrob Agents Chemother. 2018 Oct 24;62(11): 01158-18.

13. Mann D, Zipes D, Libby P, Bonow R. Braunwald a textbook of cardiovascular medicine edition 10th ed. 2014; p 1639-40.

14. Benali K, Louedec L, Azzouna BE, et al. Preclinical Validation of 99mTc-Annexin A5-128 in Experimental Autoimmune Myocarditis and Infective Endocarditis: Comparison with 99mTcHYNIC-Annexin A5. Mol Imaging 2014;13:1-10.

15. Afonso L, Kottam A, Reddy V, Penumetcha A. Echocardiography in Infective Endocarditis: State of the Art. Curr Cardiol Rep. 2017;19(12):127. 